\title{
Robotic telesurgery for achalasia
}

\author{
Kevin M. Reavis · David R. Renton · W. Scott Melvin
}

Accepted: 13 November 2006/ Published online: 20 January 2007

(C) Springer London 2007

\begin{abstract}
The craft of surgery has always relied on the use of instruments. Innovations in surgery have paralleled innovations in instrumentation. Advances in surgical instrumentation continue today and have enabled huge strides in surgical procedures and outcomes during this generation. Computers and related technology are now changing the interface between the surgeon and the patient, and are poised to improve patient outcomes by enhancing the surgeon's skills and training. The application of computer enhanced telemanipulators, or "robots", may specifically enhance operations, for example Heller myotomy, that require good visualization and precise careful dissection of delicate structures. This review covers the pathophysiology of achalasia and its history of medical and surgical treatment, leading to modern robotic telesurgical approaches. Improvements in outcome from medical to standard surgical to robotic telesurgical approaches are discussed. Current operative technique for robotic telesurgical treatment of achalasia is described and the authors conclude with a glimpse of where, in the future, current research endeavors will lead us in the treatment of achalasia.
\end{abstract}

Keywords Achalasia - Computer assisted . Heller myotomy $\cdot$ Robotic assistance

K. M. Reavis $(\varangle)$ · D. R. Renton · W. S. Melvin Division of General Surgery, The Ohio State University, RM N 703 Doan Hall, 410 W. Tenth Avenue,

Columbus, OH 43210, USA

e-mail: kevin.reavis@osumc.edu

D. R. Renton

e-mail: david.renton@osumc.edu

W. S. Melvin

e-mail: scott.melvin@osumc.edu

\section{Introduction}

Achalasia is a swallowing disorder that affects one in 100,000 individuals. Most people are diagnosed between the ages of 25 and 60 years. It initially presents with difficulty swallowing and is a chronic progressive condition that usually does not resolve [1]. Although the specific cause is unknown, the primary pathology involves the inability of the lower esophagus to relax, resulting in delayed passage of food and liquid into the stomach. Diagnosis is based on a combination of clinical symptoms and objective evaluations. Clinical symptoms range from initial dysphagia to heartburn, a feeling of fullness in the chest and sometimes weight loss. The objective work-up includes barium radiography, esophagoscopy, and esophageal manometry. In patients with achalasia barium radiography often reveals a classic "bird's beak" appearance (Fig. 1) in which the dilation tapers into the lower esophagus, because of the chronic buildup of food contents. Esophagoscopy often shows retained food and possible fermentation. Manometric evaluation reveals nonperistaltic movement within the esophagus during swallows. Long-term sequelae include an increased risk of the development of esophageal cancer in patients diagnosed with achalasia.

The objective of treatment is to resolve symptoms in this usually progressive disease. Conservative maneuvers have included the use of muscle relaxing medications or injections and dilation of the lower esophagus with endoscopically guided balloons. These usually result in temporary relief only rather than achieving long-term resolution of the problem. Surgical intervention involving splitting of the restrictive external muscle of the lower esophagus and upper 


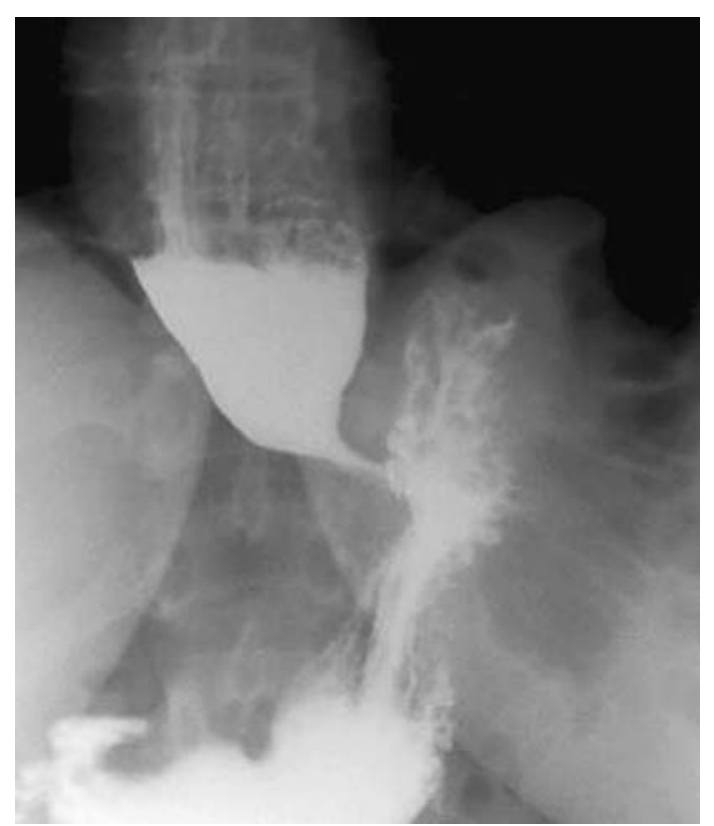

Fig. 1 "Bird's beak" appearance of lower esophagus during an upper gastrointestinal X-ray swallow study

stomach with subsequent wrapping of the upper stomach partially around the lower esophagus results in symptom relief in $85 \%$ of people 10 years after surgery and in approximately $65 \%$ of people 20 years after surgery [1].

Achalasia was first described, with its initial treatment, by Thomas Willis in 1679 [2]. Treatment and instrumentation have rapidly evolved since his first description of dilation of the esophagus using a tapered whale bone. In 1887, Russel described initial balloon dilatation using a silk bag over a rubber balloon [2]. Treatment over the last century has mainly focused on endoluminal dilatation or surgical myotomy. The first esophageal myotomy was described in 1913 by Heller. This surgical technique involved splitting the outer muscle of the lower esophagus to relieve internal narrowing and associated swallowing difficulties [3]. This procedure was subsequently popularized by approaches through the abdomen or through the chest. In the early 1990s, during the beginning of the era of minimally invasive surgery, thoracoscopic [4] and laparoscopic [5] Heller myotomy were described. Prompt recovery and good outcomes quickly enabled clinicians to conclude that surgical treatment was the optimum primary treatment of choice for amelioration of symptoms in patients with achalasia [5].

Laparoscopic Heller myotomy using standard laparoscopic techniques has evolved into an extremely safe and efficient technique of reducing symptoms in the treatment of achalasia. Preliminary case series demonstrated that early outcomes were favorable and the perioperative course was much improved compared with an open laparotomy or thoracotomy [5]. Perioperative complications were reduced and the complications secondary to the trauma of the incision were largely eliminated. Laparoscopic Heller myotomy has now been widely reported with large case series with relatively good outcomes in both early and late results [6-8]. In addition, favorable outcomes have been achieved in some series in which laparoscopic Heller myotomy was offered as the first-line therapy [9].

Although patient satisfaction, relief of symptoms, and recovery time were categorized as favorable, these large case series still reported a significant number of intraoperative esophageal perforations ranging between 1 [10] and $15 \%$ [5]. In these series, the mucosal perforations were identified to have occurred as a technical failure during the course of the division of the muscle fibers from the underlying esophageal mucosa. The clinical relevance of these complications may be uncertain, and when the perforation is recognized at the time of surgery and repair performed immediately the outcomes are still usually good. These perforations expose the patients to a prolonged hospital stay and the risk of an esophageal leak, however. Delayed diagnosis of an esophageal perforation is a potential fatal complication and is one of the most significant and serious risks of Heller myotomy. Management in this situation requires broad-spectrum antibiotic coverage, proximal bowel decompression and rest, potential operative drainage, debridement and reconstruction of the injured tissues, and sometimes intravenous nutritional support for a prolonged period of time. Different surgical techniques have been described to avoid the perforation of the underlying mucosa and have included the injection of epinephrine into the submucosal plane, blunt dissection, sharp dissection, and intraoperative esophagoscopy to evaluate the esophageal mucosa and detect underlying perforations.

\section{Operative technique and outcomes}

The da Vinci surgical system (Intuitive Surgical, Inc; Sunnyvale CA, USA) is currently the only robotic telesurgical device marketed and approved for general surgery in the United States. The device is a computer interface that interacts with the operating surgeon who sits at a control panel connected to a bedside actuator with instrument holders and a camera manipulator (Figs. 2, 3). da Vinci has instruments that rotate and articulate around three axes. Motion is enhanced by filtering fine motor tremor and providing motion scaling between the hand-piece and the activator arm, 


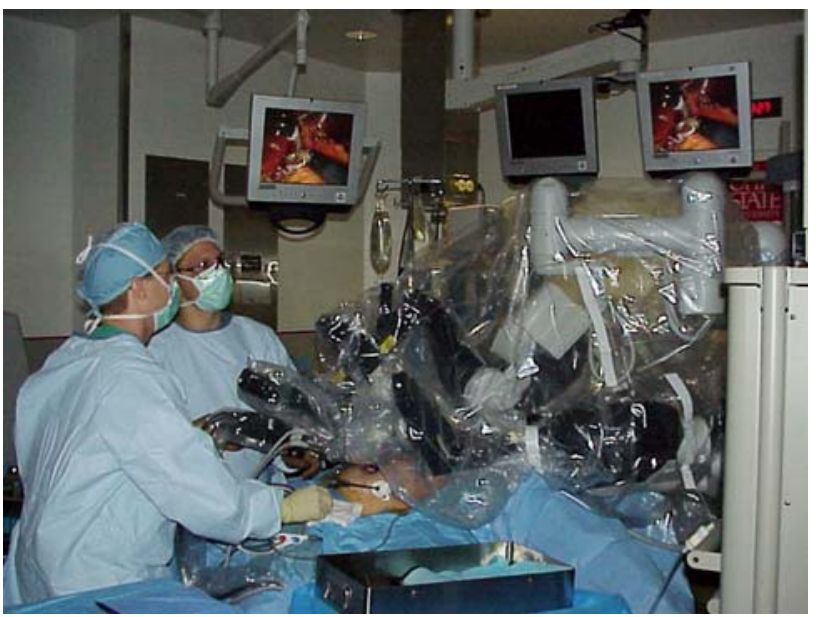

Fig. 2 The da Vinci surgical robot in use with assistant surgeons alongside operative table

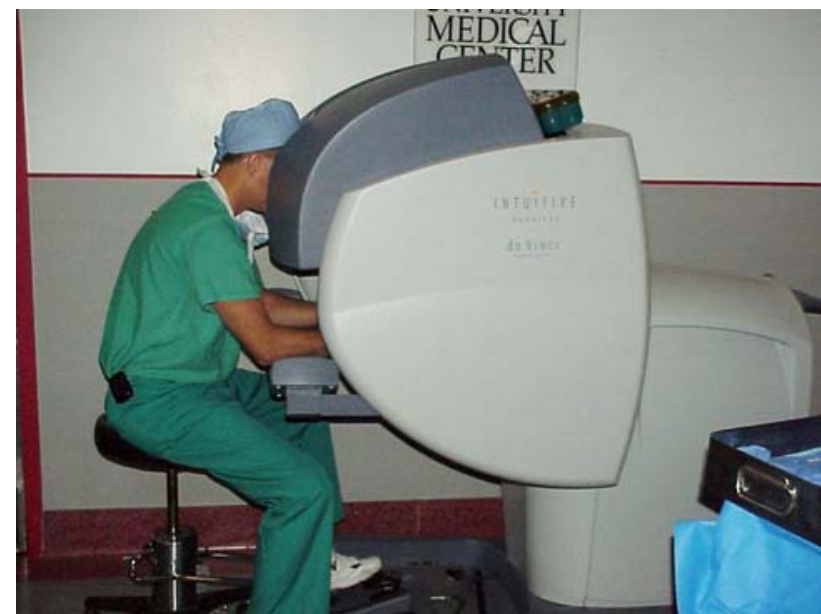

Fig. 3 The da Vinci surgical robot in use with head surgeon operating from the console

enabling up to 5-to-1 scaling. Innovations have now incorporated $5 \mathrm{~mm}$ instrumentation enabling reduced abdominal wall trauma and addition of a fourth working arm for enhanced mobility, tissue handling, and retraction.

The robotic device has the unique ability to enhance surgical technique and, therefore, optimize surgical outcome. This enhancement may make more of a difference in some surgical procedures. These advantages specifically are fine motor control, three-dimensional magnification, and motion scaling. These may be significant in operative procedures such as Heller myotomy or in cases when optimum completion of the operation requires these specific surgical techniques. It is this enhancement of surgical performance that may have improved the surgical outcomes mentioned above regarding recent case series comparing robotic telesurgery with historic laparoscopic approaches to Heller myotomy [11]. Using this model it may be reasonable to identify some surgical procedures and interventions, for example Heller myotomy, that can specifically benefit from this type of therapy. At the author's institution robotic telesurgery for achalasia is performed in the following manner.

Preoperative preparation of patients with achalasia may include a prolonged period of fasting to completely empty the esophagus. Clear liquids for $48 \mathrm{~h}$ preoperatively are routine, especially in cases of severe esophageal dilatation. Preoperative hospital admission may be necessary for patients to enable them to maintain fasting with intravenous hydration. Preoperative antibiotics and oral anti-fungal medications should be included to help reduce the amount of contamination within the esophagus. Deep venous thrombosis prophylaxis is given routinely.

The patient is positioned on a split leg table or in a modified lithotomy (legs apart and gently angled down) position after the induction of general anesthesia. The bedside component of the robot is positioned over the patients left shoulder after accessing the abdomen through four ports. The patient is placed with the head of the bed angled upwards and the patient's motion must be completed before docking to the robot ports. Two working ports are used with downward traction of the upper part of the stomach by the assistant or the fourth arm of the robot. The esophageal hiatus of the diaphragm is identified and the crura are circumferentially dissected free of peritoneal attachments. The distal esophagus is dissected, and a soft rubber Penrose drain is usually placed around the distal esophagus for traction, enabling dissection up into the lower mediastinum. The esophageal myotomy is created using the electrocautery hook of

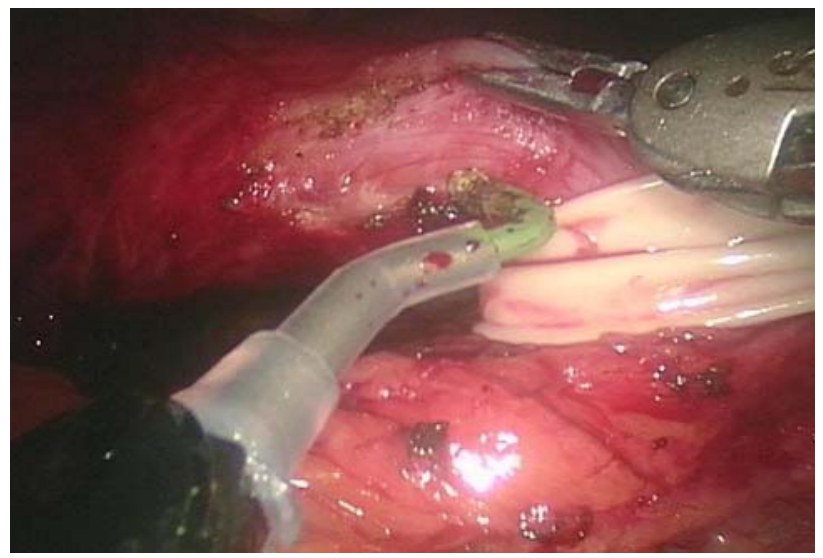

Fig. 4 Operative step of longitudinal esophageal myotomy using electrocautery. The rubber Penrose drain is used for retraction of the abdominal esophagus during this step 
the robotic device (Fig. 4). The articulation of this arm provides precise control and helps keep the end of the hook directly parallel to the esophageal mucosal wall. This dissection is aided by a curved dissector on the opposite arm, to help completely separate the muscle fibers. When the submucosal is plane is developed between the outer esophageal musculature and the inner mucosa, the division of the muscle fibers is carried $8-10 \mathrm{~cm}$ proximal and at least $2 \mathrm{~cm}$ distally from the junction of the esophagus and stomach. The gastric dissection is clearly identified by the disordered character of the vasculature of the gastric submucosa. The mucosa is carefully inspected for injury and for complete division of all muscle fibers. The muscular bundles bordering the esophageal diaphragmatic hiatus are reapproximated behind the esophagus using nonabsorbable sutures (Fig. 5). A partial gastric fundoplication is then created using a $90^{\circ}$ anterior Dor or a $270^{\circ}$ posterior Toupet fundoplication (Fig. 6). The robot is disengaged from the port sites, which are closed with sutures. Patients are allowed liquids post operatively and then are maintained on a soft diet for several days.

Postoperative patients should receive routine follow-up consisting of surveillance endoscopy every 3 years in anticipation of early detection of asymptomatic pathology. Patients who complain of recurrent symptoms should undergo formal evaluation and workup as outlined for initial patients suspected of having achalasia. Long-term postoperative results for achalasia patients are good, particularly when patients are selected with preoperative lower esophageal sphincter pressures greater than $35 \mathrm{mmHg}$ [12]. For a group of 174 patients Ruffato et al. reported overall symptomatic relief of dysphagia in $87 \%$ of patients 15 years after Heller myotomy and Dor fundoplication with $9 \%$

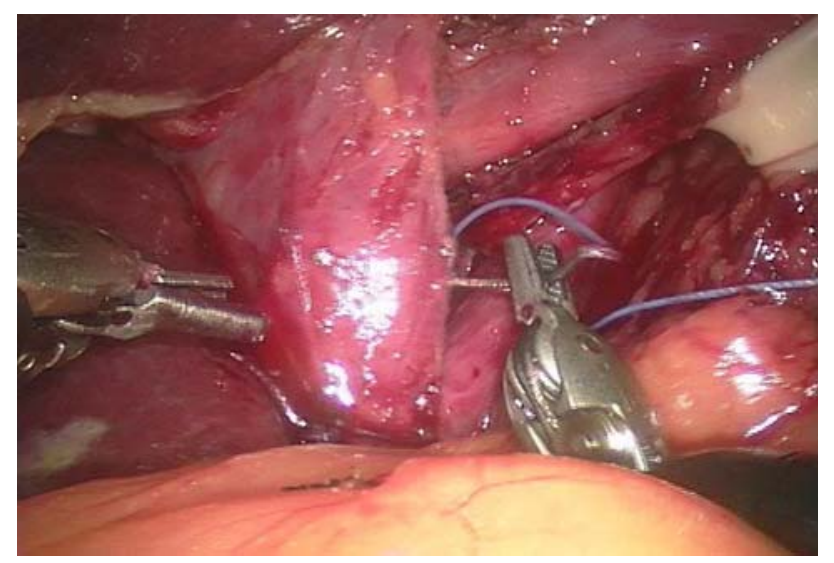

Fig. 5 Operative step of diaphragmatic posterior crural repair using robotic instrumentation. Note: the abdominal esophagus is coursing through the diaphragm in the top of the photograph of patients requiring medical/surgical treatment of symptomatic reflux and $4 \%$ of patients requiring esophagectomy for malignant disease or recurrent disease not amenable to repeat myotomy and fundoplication [13]. In patients who require re-operation for recurrent dysphagia following esophagomyotomy symptoms often present within the first postoperative year and the root cause is usually inadequate or healed myotomy. Although success can usually be achieved, results from repeat esophagomyotomy are less impressive than adequate initial operations [14]. Subtotal esophagectomy for patients with achalasia is infrequently required. It is indicated after failed medical therapy, pneumatic dilation, non-resecting surgical, and redo procedures. Gockel et al. performed subtotal esophagectomy for eight patients for whom previous therapy had failed. Dysphagia was resolved and oral alimentation was restored in each patient [15].

\section{Discussion}

Although laparoscopic Heller myotomy has emerged as the treatment of choice for achalasia, the intraoperative esophageal perforation rate of $1-15 \%$ has remained a significant problem that has prompted investigation of improvements of this operative technique. In 2000, the da Vinci surgical system (Intuitive Surgical, Inc; Sunnyvale CA, USA) a robotic telesurgical device, entered the market. The advantages reported include a stable work platform, a magnified three-dimensional image, fine motor control of articulated instruments, and motion scaling, which are uniquely designed to facilitate surgery requiring fine tissue manipulation. Disadvantages of robotic telesurgery systems include the cost of purchase and maintenance. They are large and may limit access to the patient during surgery. They provide a narrower field of view of the operative site and they provide the surgeon with essentially no tactile feedback [16]. With these advantages and hurdles in mind, a variety of surgical procedures have been reported using robotic telesurgical devices. Initial data obtained before Food and Drug Administration approval was obtained in clinical trials performing cholecystectomy and gastroesophageal fundoplication. After Food and Drug Administration approval brief clinical reports described cholecystectomy [17], fundoplication [18], colon resection [19], pancreatic resection [20], Heller myotomy [21], and other general surgery procedures [19, 22]. With experience, case series were presented which eventually demonstrated the safety and feasibility of robotic general surgery procedures [22, 23]. Reports have also been presented that look at traditional lapa- 


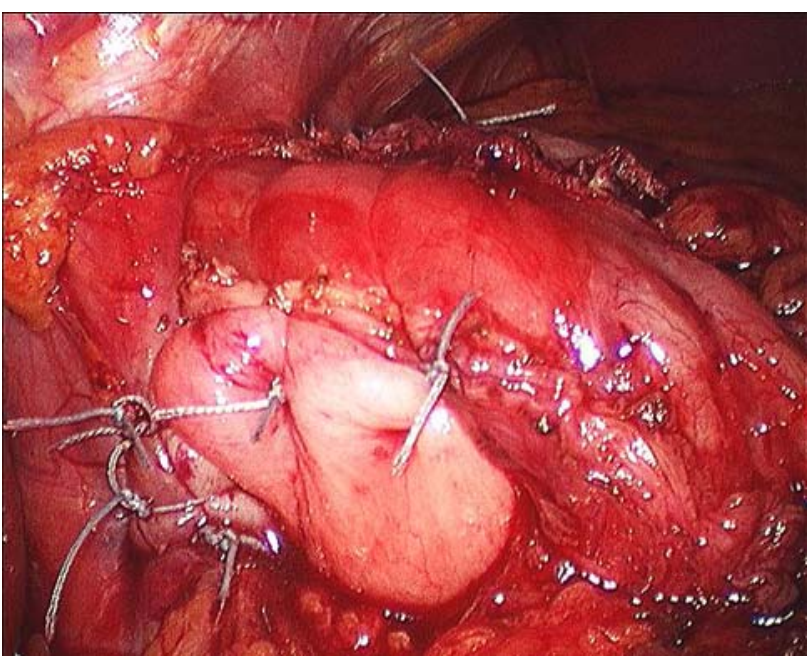

Fig. 6 Operative completion of the Heller myotomy and posterior $270^{\circ}$ Toupet fundoplication

roscopic procedures compared with robotic telesurgical assistance. Melvin et al. reported a comparison case series of gastroesophageal fundoplication performed by standard laparoscopy or with robotic telesurgical assistance [24]. Both techniques were safe and associated with low occurrence of complications; no clear benefit was seen in the robotic group, however.

Use of the da Vinci robotic telesurgical device to perform a Heller myotomy was first reported in 2001 [21] and a subsequent small case series was then reported $[23,25]$. The academic robotic surgery group, comprising surgeons from Ohio State University, Columbus, $\mathrm{OH}$, USA, Johns Hopkins University, Baltimore, MD, USA, and the University of Illinois at Chicago, Chicago, IL, USA, has prospectively collected data on 104 robotic telesurgical Heller myotomies, the largest reported case series, which were performed without a single esophageal perforation. Similar results favoring the robotic telesurgical approach were obtained in three subsequent published series comparing robotic telesugical Heller myotomy with laparoscopic Heller myotomy [26-28].

This improvement over standard laparoscopic studies which reflected the esophageal perforation rate of $1-15 \%$ may be related to the advantages of the robotic telesurgical technique and surgical enhancement the device offers compared with laparoscopic techniques [11].

\section{Future directions}

The surgical enhancement of robotic telesurgery is being applied to increasingly complex procedures. One pilot study evaluated the technique of pancreaticojejunostomy after pancreatic head resection using the robotic surgical device. Five patients were enrolled and underwent a computer-enhanced pancreaticojejunostomy using a 6-0 suture in an open abdomen after pancreatic head resection. Good results were obtained and all the anastomoses were completed [29].

Robotic assistance also has the potential to contribute to the evolving technology of natural orifice transluminal endoscopic surgery (NOTES) in which therapeutic approaches to abdominal surgical diseases are being pursued via an endoscope that is advanced from an intraluminal position within the stomach, vagina, bladder, or rectum into the abdominal cavity to perform a variety of diagnostic and therapeutic maneuvers. This was first attempted with simple peritonoscopy and has recently advanced to organ retrieval, for example appendectomy [30, 31]. Although no formal treatments for achalasia by a NOTES approach have been published, a treatment involving transgastric peritonoscopy with retroflexion and endoscopic myotomy with potential intraluminal gastroesophageal plication may prove to be feasible for treatment of achalasia with end results similar to Heller myotomy and fundoplication.

\section{References}

1. Pasricha PJ (2006) http://patients.uptodate.com/topic.asp? file $=$ digestiv/4384 September 262006

2. Brewer LA (1980) History of surgery of the esophagus. Am J Surg 139:730-743

3. Heller E (1914) Extramukose cardioplatik beim chronishcen cardiospasmus mit dilaton of the oesphagus. Mitt Grenz Med Chir 27:141-149

4. Pelligrini C, Wetter LA, Homan CB, et al (1992) Thoracoscopic esophagomyotomy: initial experience with a new approach for the treatment of achalasia. Ann Surg 216:291-299

5. Hunter JG, Trus TL, Branum GD, et al (1997) Laparoscopic heller myotomy and fundoplication for achalasia. Ann Surg 225(6):655-664

6. Heniford BT, Matthews BD, Kercher KW, et al(2001) Laparoscopic anterior esophageal myotomy and toupet fundoplication for achalasia. Am Surg 67(11):1059-1067

7. Patti MG, Fisichella PM, Perretta S, et al (2003) Impact of Esophageal Achalasia: A Decade of Change. J Am Coll Surg 196(5):698-703

8. Sharp K, Khaitan L, Scholz S, et al (2002) 100 Consecutive minimally invasive Heller myotomies: lessons learned. Ann Surg 235(5):631-638

9. Bloomston M, Serafini F, Rosemurgy AS (2001) Videoscopic Heller myotomy as first line therapy for severe achalasia. Am Surg 67(11):1105-1109

10. Finley RJ, Clifton JC, Stewart KC, et al (2001) Laparoscopic Heller myotomy improves esophageal emptying and the symptoms of achalasia. Arch Surg 136(8):892-896

11. Melvin WS, Dundon JM, Talamini M, et al (2005) Computer-enhanced robotic telesurgery minimizes esophageal perforation during Heller myotomy. Surgery 138(4):553-558 
12. Torquati A, Richards WO, Holzman MD, et al (2006) Laparoscopic myotomy for achalasia: predictors of successful outcome after 200 cases. Ann Surg 243(5):587-591

13. Ruffato A, Mattioli S, Lugaresi ML, et al (2006) Long term results after Heller-Dor operation for oesophageal achalasia. Eur J Cardiothorac Surg 29(6):914-919

14. Mercer CD, Hill LD (1986) Reoperation after failed esophagomyotomy for achalasia. Can J Surg 29(3):177-180

15. Gockel I, Kneist W, Eckardt, et al (2004) Subtotal esophageal resection in motility disorders of the esophagus. Dig Dis 22(4):396-401

16. Wright AS, Gould JC, Melvin WS (2004) Computer-assisted robotic antireflux surgery. Minerva Gastroenterol Dietol 50(3):253-60

17. Marescaux J, Smith MK, Folscher D, et al (2001) Telerobotic laparoscopic cholecystectomy: initial clinical experience with 25 patients. Ann Surg 234(1):1-7

18. Cadiere GB, Himpens J, Vertruyen M, et al (2001) Evaluation of telesurgical (robotic) Nissen fundoplication. Surg Endosc 15(9):918-923

19. Cadiere GB, Himpens J, Germay O, et al (2001) Feasibility of robotic laparoscopic surgery: 146 cases. World J Surg 25(11):1467-1477

20. Melvin WS, Krause KR, Needleman BJ, et al (2003) "Robotic" resection of a pancreatic neuroendocrine tumor. Journal of Laparoendosc Adv Surg Tech 13(1):33-36

21. Melvin WS, Krause KR, Needleman BJ, et al (2001) Computer assisted "robotic" Heller myotomy: initial case report. J Laparoendosc Adv Surg Tech A 11(4):251-253
22. Melvin WS, Krause KR, Needleman BJ, et al (2002) Computer assisted "robotic" foregut surgery, initial experience in North America. Surg Endosc 16(12):1790-1792

23. Talamini M, Chapman WC, Horgan S, et al (2003) Evaluation of 211 "robotic" surgical procedures. Surg Endosc 17:1521-1524

24. Melvin WS, Needleman BJ, Krause KR, et al (2002) Computer-enhanced versus standard laparoscopic antireflux surgery. J Gastrointest Surg 6(1):11-16

25. Melvin WS, Krause KR, Needleman BJ, et al (2002) Computer assisted "robotic" foregut surgery, initial experience in North America. Surg Endosc 16(12):1790-1792

26. Galvani C, Gorodner MV, Moser F, et al (2006) Laparoscopic Heller myotomy for achalasia facilitated by robotic assistance. Surg Endosc 20(7):1105-1012

27. Iqbal A, Hadner M, Desai K, et al (2006) Technique and follow-up of minimally invasive Heller myotomy for achalasia. Surg Endosc 20(3):394-401

28. Horgan S, Galvani C, Gorodner MV, et al (2005) Robotic assisted Heller myotomy versus laparoscopic Heller myotomy for the treatment of achalasia: a multicenter study. J Gastrointest Surg 9(8):1020-1029

29. Melvin WS, Mikami DJ, Gould JC, et al (2003) Computer enhanced pancreaticojejunostomy following pancreaticoduodenectomy. Surgical Endoscopy 17(Suppl 1):S301

30. Ponsky JL (2005-2006) New frontiers in endoscopic surgery. Personal notes

31. Swanstrom LL, Kozarek R, Pasricha PJ (2005) Development of a new access device for transgastric surgery. J Gastrointest Surg 9(8):1129-1136 